\title{
SURGICAL ASPECTS OF SPINAL CONDITIONS
}

By HaRVEY Jackson, F.R.C.S.

Hon. Neurological Surgeon, St. Thomas's Hospital ; Hon. Surgeon, The National Hospital, Quee Square ; Consultant Neurosurgeon, Ministry of Pensions Hospital, Roehampton

\section{Surgical Treatment in Spinal Conditions}

Unfortunately such is the prognosis of neurological disorders that should surgical intervention not be applicable, there is little to offer in the hope of cure, or even alleviation of the patient. Consequently, it behoves one to be constantly on the look-out for evidence of distortion, friction pressure or other mechanical factor likely to be playing at least some part in the production of symptoms. So the field of surgery of the spinal cord has extended in the alleviation of incapacity arising out of diseases both of the cord and of other structures. Surgical relief may be applied to pain, tremor, spasm and deformity. In so far as cord lesions are concerned we see surgical indications in tumour formation, arachnoiditis, syringomyelia, traumatic effects and even the cure of sciatica.

\section{Spinal Compression}

It is paramount, of course, in the treatment of any patient that an acurate diagnosis be reached. In no state has improvement along these lines been more apparent than in the problem of Spinal Compression. This vague term has been applied to such a diversity of states, e.g. Paget's Disease or other generalized bone disease involving the spine, epidural spinal abscess, intraspinal neoplasms of either intramedullary or extramedullary situations, etc., yet each and all of these should formulate a clinical syndrome whereupon an accurate diagnosis should be possible. Meanwhile, one must realize that the symptomatology is to be explained not on one mode of production but on several different factors. It may arise out of interference with the blood supply by the effect of pressure directed to the blood vessels or through vascular stasis and throm- bosis, or as the result of failing neuronat conductivity. Full appreciation of these facts bears on an estimation of the prognosis in given case; whereas direct mechanica $\mathrm{B}$. pressure brings about some reversible changes $\tilde{c}_{\tilde{N}}$ a vascular thrombosis offers a poorer prospect心 of equivalent available recovery. Indeed, ino virtue of the former process most remarkable functional restoration attends successful re $\mathrm{re}_{0}^{+}$ moval of an extramedullary tumour, in spite of the fact that the spinal cord has sufferes such severe compression as to have assume almost ribbon-like dimensions.

Arachnoiditis. Attention has bein directed of recent years to the condition Arachnoiditis. A literal interpretation to the term Arachnoiditis is somewhat mis leading for the meningeal involvement is onlyo part of the attendant pathology, associated changes of vascular or parenchymatous origirs invariably take part. As the proportions of meningeal and cord involvement vary fromp case to case, so we find different reactions to treatment. Occasionally the disorder is almose restricted to the meninges, in fact the result og the disorder becomes so localized as to produce loculation of the subarachnoid space, perhaps to form a single loculus (Meningitis Serosæ Circumscripta). When operation reveals this isolated lesion, the prognosis is correspondingle improved. Should the meningeal reaction be diffuse, or should profound vascular softening? obtrude, then the chances of appreciable improvement become minimized. At times one finds that as a result of acute meningitis: the cord is softened and degenerated to the extent of recognizable structure being comen pletely lacking. Whilst a single cyst may arise from an acute meningitis, it is much more commonly of developmental origin. Cysts o 
developmental origin may be situated outside the dura mater too, when a polycystic variety is more common than the isolated loculus. That all cases of Arachnoiditis originate in acute disease would be a false conception; in fact, many cases are of a chronic nature from the start. What factors of causation lie behind this latter process is not as yet determined, but of trauma, toxaemia, alcoholism, syphilis, and a number of other conditions, each plays some part in one case or another.

Spinal tumours. One cannot, of course, discuss the surgery of spinal conditions without considering tumour formation. So wide a subject is this, however, that adequate reference cannot be devoted to the pathology, symptomatology and therapy in an article of this character. A few outstanding important points must be mentioned and the reader referred to text-books for general detail.

The compressive syndrome, which, after all, is that state wherein tumour formation is to be recognized, evidences itself in clinical phenomena indicative of a lesion of progressive development at some recogniżable neurological level. The clinical evidence gains confirmation perhaps in radiological examination, perhaps in evidence of obstruction within the cerebrospinal fluid pathways as indicated by the Queckenstedt test. A loculation syndrome (Froin's Syndrome) may occur in a concentration of the total protein content of the cerebrospinal fluid, caudal to the obstruction.

The importance of pain. In a major proportion of tumour cases, pain plays an important part. The pain is not of one type, moreover the characteristics of the pain bear significant identification, e.g. a tumour of a vertebra exhibits that type of pain commonly associated with bone disease in general, a pain of a constant, dull, boring nature, that is subject to variation with atmospheric changes. A neurofibroma growing from a posterior nerve root induces a sharp, cutting type of pain of a distribution coinciding with the course of the affected nerve; a pain that is liable to accentuation on coughing, sneezing or straining. Yet a third type of pain occurs in spinal cord affections, as in some other more central disorders-so-called 'central pain.' This is of a constant, aching or burning quality, finding reference to the more paretic side, incidentally the side of the lesser sensory change in the typical Brown-Sequard lesion. In tumours involving the cauda equina, pain is commonly of root distribution and involves areas of depressed sensory perception, although simple clinical examination may fail to reveal definite objective sensory loss.

The pain derived from a neurofibroma of a posterior root may be so intensified by the slightest movement that the patient fears the approach of anyone towards the bed, lest the slightest jar set off an attack. Such is the intensity of pain that this alone can be applied to differentiate a tumour from some other cause of root pain, e.g. a disc protusion. One must not assume, therefore, that a neurofibroma essentially causes such root pain. In fact, massive tumours or 'giant tumours' of the lumbar roots sometimes develop either within or outside the dura, to cause persistent and very chronic backache as the sole symptom, extending over a period of years. Patients so afflicted are to be seen with marked pigmentation of the back due to prolonged physiotherapy. The diagnosis is commonly delayed until gross dimensional changes in the spinal canal become evident on X-ray examination.

Secondary deposits. Due appreciation of the incidence of metastatic malignant deposits in the spine is lacking, and consequently many sad cases fail to be recognized. Certainly of all the forms of spinal compression, secondary deposits are commonest, being responsible for something in the order of 20 to 25 per cent. of cases. Sites of selection are to be noted, and these can be indicative of the source in the search for a primary focus. For instance, the upper four or five dorsal vertebrae are most often selected by metastases of breast origin; the mid-dorsal vertebrae tend to be involved by secondaries from the lung, whilst the lower dorsal and more caudal vertebrae are prone to be affected when the primary lies in the prostate. Although these are the more common origins of secondaries in the spinal column, carcinoma of other organs (thyroid, suprarenal, gastrointestinal tract, etc.) may metastasize in the spine, as in other bones. Not infrequently a malignant focus in the lung fails to be located, then the diagnosis may remain in doubt. Yet typical 
bone pain, rapid deterioration, and the development of a complete motor and sensory paraplegia make the diagnosis of malignant disease almost unchallengeable. That this is invariably the picture of malignant disease would be a false impression, as persistent localized pain has been known to symptomatize secondary deposits in the spine over periods of twelve months or even longer, in the absence of neurological manifestations.

Inflammatory disorders. Something must be added to the limited reference to inflammatory disorders in their causation of paraplegia. The acute pyogenic, epidural spinal abscess is appreciated generally, more pehaps, than the chronic granuloma. Unfortunately, the diagnosis of an acute abscess in such a situation is not commonly made until paralytic phenomena are obvious. When the diagnosis should be reached, if full benefit is to accrue from treatment, is during the preparetic stage. This means that the existence of the abscess should be realized when a patient is afflicted by intense pain in the back, perhaps with associated root pain, is in a febrile state, and gives a history of some recent septic infection, e.g. boil, carbuncle, whitlow, sore throat, alveolar abscess, etc. Usually the spinous processes of the levels involved are very tender, possibly to touch, certainly on percussion with the finger.

Just where the infective embolus becomes arrested in the formation of the abscess is of interest. Though it is possible for the condition to arise out of an osteomyelitic focus, rather is it due to a direct metastatic lesion of the epidural tissues-that cushion of fatty tissue lying between the bony canal and the dura mater.

In the patient with paralytic changes the effect of laminectomy is commonly disappointing. The relief from pressure so effected is not met by recovery; a matter calling attention to the pathological basis of the attendant paralysis. Obviously compression alone is not responsible. As the course of the paralytic development is so fulminating, it is probably brought about by spreading vascular thrombosis. The prognosis in these cases is poor, but the advent of penicillin has greatly improved the prospects of control of the infective process, with a reduction in the risk of attendant thrombosis. Nevertheless, penicillin administration is not to be looked upon as a means of avoiding surgery, otherwise, even should the patient live, paralytic sequelae are bound to result It has been possible to modify the surgical procedure by the use of penicillin, the laminectomy need not be so extensive, and it is not necessary for the wound to be left completely open.

An important matter in the clinical investigation of a case of suspected epidural abscess is the avoidance of lumbar puncture owing to the attendant risk of septic meningitis.

\section{Other Spastic States}

Syringomyelia. Apart from new growths, indeed, in some cases, in consequence of a new growth, the state of syringomyelia may develop in the cervical or dorsal segments of the spinal cord. Usually a slowly progressive weakness of the upper limbs occurs together with sensory disturbance of a dissociated type. Later, as distortion of the cord in $\overrightarrow{0} \overrightarrow{0}$ creases, spastic weakness of the lower limb? develops. The paretic state may be tardy in development, the patient then coming undep observation perhaps because of a painless, infective lesion of the hand, or possibly owing to the occurrence of a Charcot's joint in one of the articulations of the upper limb. Should extension of the process give rise to a spastic paraplegia of incapacitating degree, the possibility of a compressive state calls for investigation in the form of radiographic examination of the spine and lumbar puncture with cerebrospinal fluid analysis. Not infrequently with the extension of cavitation within the cord, the lesion tracts posteriorly towards the cord surface ; in so doing it brings about a reduction in position-sense and vibration-sense in the lower limbs. This change further supports the feasibility of aid from surgical intervention, for then the necessary incision into the cord will not add to the risk of increased disability from damage to the posterior columns of the cord.

No matter how satisfactory surgical intervention may prove in these patients, the application of deep X-ray therapy as a postoperative measure is advisable. 
Scoliosis. Sometimes an increasing spasticity is the outcome of spinal deformity, occasionally from a skeletal change in a pre-existent lesion. This is to be seen in Adolescent Scoliosis. A child with a scoliosis of congenital nature, or possibly the sequel to Poliomyelitis, on reaching the age of adolescence, undergoes a rapid deterioration in that the deformity becomes exaggerated and a spastic paraplegia ensues. Posterior column function becomes disturbed although the spinothalamic tracts are involved in intensities varying from case to case. Lumbar puncture shows a partial or complete manometric block. Exploratory laminectomy in such a patient reveals a dura mater tautly extended at the height of the vertebral curve. On incision of the dura mater the spinal cord prolapses through the dural gap, whilst the margins of the dura retract. When the cord first appears the sub-arachnoid space is seen to be collapsed; but, with the release of tension, the cerebrospinal fluid is then seen to seep through the space, surrounding the cord with a fluid-distended arachnoid.

Technically, in a case of gross scoliosis, the laminectomy is a little complicated. Approach is not easy owing to the deformity, also the spinal roots tend to assume a rather spiral arrangement as they pass from the dura to their respective exits through the intervertebral foramina.

A clinical improvement usually shows early indication.

Spinal injuries. Traumatic spastic paraplegia. Dealing with the problem of disablement from spasticity does not invariably demand intervention designed to restore motor power in the affected limbs, it may be a matter of correcting deformity. We are only too aware of the difficulties in traumatic paraplegia arising out of the position of acute flexion brought about by the spastic state of the muscles. The awkward posture, the contractures, and the reflex function preclude any prospect of mobility.

With the number of spinal injuries of wartime still freshly in mind, one is stimulated into doing something worthwhile. An important factor in instilling hope into these unfortunates is the attitude of the attendant surgeon. The demeanour of hopelessness left by the 1914-1 8 war needs adjustment, and has been replaced by an enthusiasm directed towards the production of ambulatory patients. An attitude of activation from the earliest stages can do much in the prevention of contractures, and in controlling urinary sepsis. What is to be done for these calamities brought about by penetrating wounds, fractures and dislocations accompanied by global paraplegia ? To understand the patient's burden is the first requirement, then the application of suitable aid. The difficulties are those of : immobility, spasticity, flexor spasms, contractures, active mass reflexes, urinary incontinence and bedsores. The maintenance of mobility in all joints from the earliest possible moment, together with the restoration of activity in recovering muscles by properly designed exercises are important routines. The acquisition of powerful muscle groups applied to stability and an erect posture necessitates particular attention to the erector spinae and abdominal muscles. Some patients acquire a hyperactive mass reflex which, together with spasticity and deformity, renders any prospect of mobility impossible. Relief under these circumstances requires dispersal of the spasticity with consequent avoidance both of flexor spasms and recurrent urinary incontinence. Operative intervention proved of little avail until the description by Munro ${ }^{1}$ of the relief to be obtained by the conversion of a spastic into a flaccid state. By section of the anterior roots of consecutive nerves from the eleventh dorsal to the first sacral the spasticity can be abolished, the mass reflex prevented, and the bladder given relative control. Thus the reduction of the deformity will enable suitable calipers to be applied and allow of an erect posture. The prevention of repeated urinary incontinence enables the skin to be maintained in good condition. By these means patients can be made ambulatory and, in many cases, able to engage in employment.

\section{Protruded Intervertebral Disc}

For many years, neurosurgeons have been removing pieces of extruded cartilage from the spinal canal for relief of compression of lumbosacral roots. The tissues removed proved histologically to consist of cartilage, and was usually considered to be neoplastic. In 1934, Mixter and Barr correlated the presence of such protrusions with cases of 
sciatica, thereby establishing the relationship. From that time interest anew has been devoted to the subject, but the true nature of the protusion, e.g. whether it is a rupture, hernia, or prolapse, remains somewhat obscure. Certainly, the presence of a disc protrusion is the commonest single cause of sciatica, but it is not the only cause, nor is sciatica an essential item in the diagnosis of a protrusion. As the lesion is primarily a disorder of the back, obviously evidence of abnormality must be present in the back. Although alteration of contour and mobility of the back are essential, no pain need be referable thereto. Nevertheless, a history of recurrent attacks of lumbago or fibrositis is to be elicited in the majority of patients, even though direct questioning may be necessary to its citation. The sequence of events is not always the same, for, whilst more cases are initiated with pain in the back, it is not rare to find a patient in whom the sciatica proves to be the original symptom. A most important detail in the clinical history is an intermittency or remittency in the symptomatology. In so far as the sciatic element is concerned, pain may be the only evidence, but a positive Laségue's sign is valuable conflrmation. Usually, one prefers to see some additional root disorder, sensory, motor or reflex, and that to occur in the realm of the fifth lumbar, or first sacral root. That these two roots are mentioned in particular indicates that at two levels only are disc protrusions likely to be encountered in the lumbsacral region; the level of the fourth and fifth lumbar discs. Just which level is involved can usually be judged with some accuracy on the clinical manifestations. Thus evidence, sensory or motor, of implication of the fifth lumbar root indicates that the fourth lumbar disc is the site of the trouble (disc between fourth and fifth lumbar vertebrae). The inference of a depressed or suppressed ankle jerk is this : should the accompanying signs be purely of first sacral root distribution, then the lesion lies at the fifth lumbar disc; but evidence of fifth lumbar root involvement applies as above.

The pathology of the protrusion is not really ascertained. Likewise the causation remains in doubt. There is a history of an injury in so many cases that this cannot be ignored, yet the injury is so often of such a restricted nature that it cannot account adequately for the distortion that must be involved. Many patients, of course, deny that any injury has even been sustained; others find the onset of the trouble coincident with an acute febrile illness in which they are restricted to bed. A peculiar tendency to the onset of symptoms during pregnancy is no uncommon finding, in fact disc protrusion is recognized as the cause of certain cases of obstetrical paralysis.

One must not be led to believe protrusion of the intervertebral disc to be restricted to the lumbar region, for other regions of the spinal column also are liable. However, the dorsal disc protrusion does not commonly manifest itself in quite the same manner, rather does it present clinically as a severe spinal compression, most often between the ages of 40 and 60 years.

Cervical protrusions simulate the lumbar protrusions in their tendency to afford evidence of root disturbance, also they give a similar history of injury in many cases. Again the injury proves to be of minor nature, and more of an aggravating than an inciting factor.

The clinical assumption of the presence of disc protrusion has now reached such accuracy that little verification seems necessary. Radiographic examination, nevertheless, is important even if applied merely in exclusion of unsuspected bone disease. Contrast radiology (using air, lipiodol, pantopac) was much employed, and abused, at one juncture, but has been given up by many experienced neurosurgeons. Not infrequently one sees a collapse of an intervertebral space on the radiograph; should this coincide with the level indicated by clinical findings, well and good, otherwise its value should be suspect.

Having reached the diagnosis of a protrusion what procedure is to be adopted ? Speaking generally, operative measures are not to be applied until after prolonged conservative treatments have been given due trial, and then only if incapacity is present. Conservative measures worthy of trial are : radiant heat, short-wave diathermy, plaster jacket, epidural injections of novocaine in saline, manipulation.

REFERENCE

1. MUNRO (1945), New England F. Med., 233, 453-46 r. 